\title{
An Overview of the Research on Plant Leaves Disease detection using Image Processing Techniques
}

\author{
Ms. Kiran R. Gavhale ${ }^{1}$, Prof. Ujwalla Gawande ${ }^{2}$ \\ ${ }^{1}$ Department of Computer Technology, \\ Yeshwantrao Chavan College of Engineering, Nagpur, Maharashtra, India. \\ ${ }^{2}$ Asst. Prof. Department of Computer Technology, \\ Yeshwantrao Chavan College of Engineering, Nagpur, Maharashtra, India
}

\begin{abstract}
Diseases in plants cause major production and economic losses as well as reduction in both quality and quantity of agricultural products. Now a day's plant diseases detection has received increasing attention in monitoring large field of crops. Farmers experience great difficulties in switching from one disease control policy to another. The naked eye observation of experts is the traditional approach adopted in practice for detection and identification of plant diseases. In this paper we review the need of simple plant leaves disease detection system that would facilitate advancements in agriculture. Early information on crop health and disease detection can facilitate the control of diseases through proper management strategies. This technique will improves productivity of crops. This paper also compares the benefits and limitations of these potential methods. It includes several steps viz. image acquisition, image pre-processing, features extraction and neural network based classification.
\end{abstract}

Keywords: Disease detection, Image acquisition, pre-processing, features extraction, classification, symptoms and neural network.

\section{Introduction}

India is an agricultural country wherein most of the population depends on agriculture. Research in agriculture is aimed towards increase of productivity and food quality at reduced expenditure, with increased profit. Agricultural production system is an outcome of a complex interaction of soil, seed, and agro chemicals. Vegetables and fruits are the most important agricultural products. In order to obtain more valuable products, a product quality control is basically mandatory. Many studies show that quality of agricultural products may be reduced due to plant diseases. Diseases are impairment to the normal state of the plant that modifies or interrupts its vital functions such as photosynthesis, transpiration, pollination, fertilization, germination etc. These diseases are caused by pathogens viz., fungi, bacteria and viruses, and due to adverse environmental conditions. Therefore, the early stage diagnosis of plant disease is an important task [1]. Farmers require continuous monitoring of experts which might be prohibitively expensive and time consuming. Therefore looking for fast [2], less expensive and accurate method to automatically detect the diseases from the symptoms that appear on the plant leaf is of great realistic significance. This enables machine vision that is to provide image based automatic inspection, process control and robot guidance. The objective of this paper is to concentrate on the plant leaf disease detection based on the texture of the leaf. Leaf presents several advantages over flowers and fruits at all seasons worldwide [3], [4].

This paper is organized into the following sections. Section 1 gives an introductory part includes importance of leaf disease detection, plant leaves analysis, various types of leaf diseases and its symptoms. Section 2 presents a detailed discussion on recent work carried out in this area. Section 3 includes basic methodology for leaves disease detection which represents a brief review on various image processing techniques. Finally, section 4 concludes this paper along with possible future directions.

\subsection{Plant diseases analysis and its symptoms}

The RGB image feature pixel counting techniques is extensively applied to agricultural science. Image analysis can be applied for the following purposes:

1. To detect plant leaf, stem, and fruit diseases.

2. To quantify affected area by disease.

3. To find the boundaries of the affected area.

4. To determine the color of the affected area

5. To determine size \& shape of fruits.

Following are some common symptoms of fungal, bacterial and viral plant leaf diseases. 


\subsubsection{Bacterial disease symptoms}

The disease is characterized by tiny pale green spots which soon come into view as water- soaked. The lesions enlarge and then appear as dry dead spots as shown in figure 1(a), e.g. bacterial leaf spot have brown or black water-soaked spots on the foliage, sometimes with a yellow halo, generally identical in size. Under dry conditions the spots have a speckled appearance.

\subsubsection{Viral disease symptoms}

Among all plant leaf diseases, those caused by viruses are the most difficult to diagnose. Viruses produce no telltale signs that can be readily observed and often easily confused with nutrient deficiencies and herbicide injury. Aphids, leafhoppers, whiteflies and cucumber beetles insects are common carriers of this disease, e.g. Mosaic Virus, Look for yellow or green stripes or spots on foliage, as shown in figure 1(b). Leaves might be wrinkled, curled and growth may be stunted.

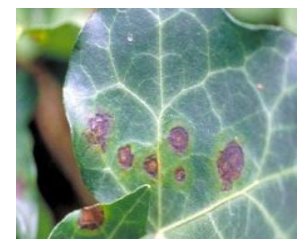

(a) Bacterial leaf spot

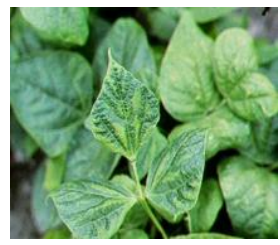

(b) mosaic virus

Figure 1. Bacterial and Viral disease on leaves

\subsubsection{Fungal disease symptoms}

Among all plant leaf diseases, those caused by fungus some of them are discussed below and shown in figure 2, e.g. Late blight caused by the fungus Phytophthora infesters shown in figure 2(a). It first appears on lower, older leaves like water-soaked, gray-green spots. When fungal disease matures, these spots darken and then white fungal growth forms on the undersides. Early blight is caused by the fungus Alternaria solani shown in figure 2(b). It first appears on the lower, older leaves like small brown spots with concentric rings that form a bull's eye pattern. When disease matures, it spreads outward on the leaf surface causing it to turn yellow. In downy mildew yellow to white patches on the upper surfaces of older leaves occurs. These areas are covered with white to greyish on the undersides as shown in figure 2(c).

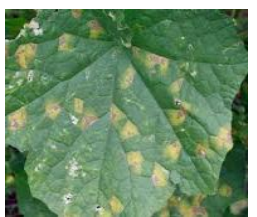

(a) late blight

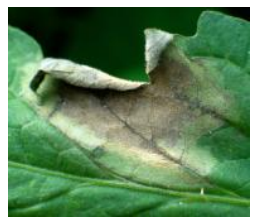

(b) early blight

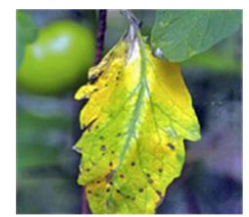

(c) downy mildew

Figure 2. Fungal disease on leaves

\section{Literature review}

A Proliferation of literature is available in plant leaf disease detection. We will highlight some of the key contributions.

A methodology for detecting plant diseases early and accurately using diverse image processing techniques has been proposed by Anand H.Kulkarni et al. [1], where Gabor filter has been used for feature extraction and ANN based classifier has been used for classification with recognition rate up to 91\%. F. Argenti, et al. [2] proposed a fast algorithm for calculating parameters of co-occurrence matrix by supervised learning and maximum likelihood method for fast classification. Homogenize techniques like sobel and canny filter has been used to identify the edges by P.Revathi et al. [3]. These extracted edge features have been used in classification to identify the disease spots. The proposed homogeneous pixel counting technique for cotton diseases detection (HPCCDD) algorithm has been used for categorizing the diseases. They claim the accuracy of 98.1\% over existing algorithm. Tushar H Jaware et al. [4] proposed a novel and improved k-means clustering technique to solve low-level image segmentation. Spatial gray-level dependence matrices (SGDM) method has been used for extracting statistical texture features by Sanjay B. Dhaygude et al. [5]. RGB images have been converted into Hue Saturation Value (HSV) color space representation and showed the H, S and V components. Mokhled S. Al-Tarawneh [6] presented an empirical investigation of olive leaf spot disease using auto-cropping segmentation and fuzzy c-means classification. Rgb to Lab colorspace and median filter used for image enhancement. At end present comparative assessment of fuzzy c-means and k-mean clustering. 
The fuzzy feature selection approach namely fuzzy curves (FC) and fuzzy surfaces (FS) have been proposed to select features of cotton leaf disease by Yan-Cheng Zhang, et al. [7]. This has been resulted in reduced dimensional feature space. Back-propagation (BP) networks have been used to classify the grape and wheat diseases by Haiguang Wang et al. [8]. Also by using principal component analysis (PCA) dimensions of the feature data has been reduced. Texture features based on the local power spectrum of Gabor filters has been proposed by Simona E. Grigorescu et al. [9] where complex moments, Gabor energy and grating cell operator features have been discussed. They concluded that grating cell operator responded only to texture features.

Detection of unhealthy region and classification using texture features has been proposed by $\mathrm{S}$. Arivazhagan, et al. [10]. Their algorithm has been tested on ten species of plants namely banana, beans, jackfruit, lemon, mango, potato, tomato and sapota. 94.74\% accuracy has been achieved by Support vector machine (SVM) classifier.

Dheeb Al Bashish, et al. [11] developed neural network classifier based on statistical classification and could successfully detect and classify the diseases with a precision of around $93 \%$. A Research of maize disease image recognition of corn based on BP networks effectively identified by Song Kai et al. [12] where YCbCr color space technology is used to segment disease spot, Co-occurrence matrix (CCM) spatial gray level layer is used to extract disease spot texture feature, and BP neural network has been used to classify the maize disease. The applications of K-means clustering, BP neural networks had been formulated for clustering and classification of diseases that affect on plant leaves by H. Al-Hiary, et al. [13]. They provide adequate support for accurate detection of leaf diseases. The proposed algorithm has been tested on five diseases viz. Early and late scorch, cottony and ashen mold, tiny whiteness. Menukaewjinda et al. [14] tried another ANN, i.e. back propagation neural network (BPNN) for efficient grape leaf color extraction with complex background. They also explore modified self organizing feature map (MSOFM) and genetic algorithm (GA) and found that these techniques provide automatic adjustment in parameters for grape leaf disease color extraction. Support vector machine (SVM) has been also found to be very promising to achieve efficient classification of leaf diseases. 21 color, 4 shape and 25 texture features has been extracted by Haiguang Wang et al. [15] and principal component analysis (PCA) has been performed for reducing dimensions in feature data processing, then back-propagation (BP) networks, radial basis function (RBF) neural networks, generalized regression networks (GRNNs) and probabilistic neural networks (PNNs) has been used as the classifiers to identify diseases.

\section{Methodology}

There are five main steps used for the detection of plant leaf diseases as shown in fig.3. The processing scheme consists of image acquisition through digital camera or web, image pre-processing includes image enhancement and image segmentation where the affected and useful area are segmented, feature extraction and classification. Finally the presence of diseases on the plant leaf will be identified.

In the initial step, RGB images of leaf samples were picked up. The step-by-step procedure as shown below:

1) RGB image acquisition;

2) convert the input image into color space;

3) Segment the components;

4) obtain the useful segments;

5) Computing the texture features;

6) Configuring the neural networks for recognition.

3.1. Image acquisition

Firstly, the images of various leaves acquired using a digital camera with required resolution for better quality. The construction of an image database is clearly dependent on the application. The image database itself is responsible for the better efficiency of the classifier which decides the robustness of the algorithm.

\subsection{Image pre-processing}

In the second step, this image is pre-processed to improve the image data that suppress undesired distortions, enhances some image features important for further processing and analysis task. It includes color space conversion, image enhancement, and image segmentation. The RGB images of leaves are converted into color space representation. The purpose of the color space is to facilitate the specification of colors in some standard accepted way. RGB images converted into Hue Saturation Value (HSV) color space representation. Because RGB is for color generation and his for color descriptor. HSV model is an ideal tool for color perception. Hue is a color attribute that describes pure color as perceived by an observer. Saturation termed as relative purity or the amount of white light added to hue and value means amplitude of light. After the color space transformation process, hue component used for further analysis. Saturation and value are dropped since it does not give extra information [5]. 


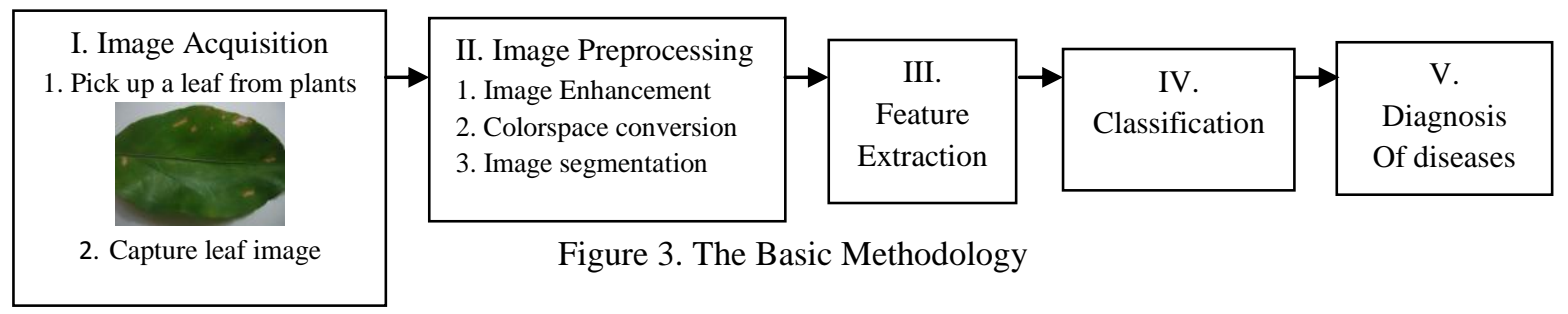

Ycbcr color system is a common color space, which is applied by the most widely used jpeg image. Y, $\mathrm{cb}$ and $\mathrm{cr}$, indicates a luminance component and two color component signals respectively. Different from other color space, Ycbcr color space is orthogonal, which fully takes important factors of composition of RGB from other colors into account. Ycbcr color space model is often used in image compression [12]. A, $\mathrm{U}$, and $\mathrm{Cr}$ components from LAB [6], UVL, and Ycbcr color space used to extract affected leaf color with the purpose of less illumination effects [14].

Image segmentation is process used to simplify the representation of an image into something that is more meaningful and easier to analyze. As the premise of feature extraction and pattern recognition, image segmentation is also the fundamental approaches of digital image processing. There are various techniques for image segmentation discuss below.

\subsubsection{Region based}

In this technique pixels that are related to an object are grouped. The area that is detected for segmentation should be closed. Due to missing edge pixels in this region based segmentation there won't be any gap. The boundaries are identified for segmentation. In every step at least one pixel is related to the region and is taken into consideration. After identifying the change in the color and texture, the edge flow is converted into a vector. Then these edges are detected for further segmentation.

\subsection{2. $\quad$ Edge based}

Segmentation can also be done by using edge detection techniques. There are various techniques viz. gradient, log, canny, sobel, laplacian, robert. In this technique the boundary is identified to segment. Edges are detected to identify the discontinuities in the image. For classification they use both fixed and adaptive feature of support vector machine.

\subsubsection{Threshold based}

It is the easiest way of segmentation. Here segmentation is done through the threshold values obtained from the histogram of those edges of the original image. So, if the edge detections are accurate then the threshold too. Segmentation through thresholding has fewer computations compared to other techniques. The disadvantage of this segmentation technique is not suitable for complex images.

\subsubsection{Feature based clustering}

Segmentation is also done through Clustering [8]. The image is converted into histogram and then clustering is done on it. Pixels of the color image are clustered for segmentation using an unsupervised technique Fuzzy C. This is applied for ordinary images. It results to fragmentation if it is a noisy image. A basic clustering k-means algorithm is used for segmentation in textured images [6,13]. It clusters the related pixels to segment the image. Segmentation is done through feature clustering and there it will be changed according to the color components. Segmentation is also purely depending on the characteristics of the image. Features are taken into account for segmentation. Difference between the intensity and color values are used for segmentation. Improved k-mean used to solve low-level image segmentation [4].

For segmentation of color image use of fuzzy clustering technique is to iteratively generate color clusters using fuzzy membership function in color space regarding to image space. The technique is successful in identifying the color region. Real time clustering based segmentation. A Virtual attention region is captured accurately for segmentation. Image is segmented coarsely by multithresholding. It is then refined by fuzzy cmeans clustering [6]. It applied to any multispectral images is a major advantage [7].Segmentation approach for region growing is k-means clustering. A Clustering technique for image segmentation is done with cylindrical decision elements of the color space. The surface is obtained through histogram and is detected as a cluster by thresholding. By using modified self organizing feature map (MSOFM), clustering process does not require predefined number of color group. This is also adjustable allowing similarity of each color group. The suitable color group numbers lead to the better color extraction [14]. 


\subsubsection{Model based}

Markov Random Field (MRF) based segmentation is known as model based segmentation. An inbuilt region smoothness constraint is presented in MRF which is used for color segmentation. MRF is combined with edge detection for identifying the edges accurately [4].

\subsection{Feature extraction}

After segmentation the area of interest i.e. diseased part extracted. In the next step, significant features are extracted and those features can be used to determine the meaning of a given sample. Actually, image features usually includes color, shape and texture features. Currently most of the researchers targeting plant leaf texture as the most important feature in classifying plants. With the help of texture features, plant diseases are classified into different types. There are various methods for feature extraction as discussed below.

\subsubsection{Texture analysis methods}

Textures are a pattern of non-uniform spatial distribution of differing image intensities, which focus generally on the individual pixels that make up an image. Texture is termed as quantifying the spatial relationship between materials in an image. Some properties are playing an important role in recitation of texture viz. uniformity, regularity, density, linearity, directionality, roughness, coarseness, phase and frequency. The method to characterize texture force into four major categories such as statistical, structural, fractals, and signal processing.

Statistical: Statistical type includes grey-level histogram, grey-level co-occurrence matrix, auto-correlation features, and run length matrices for texture extraction.

Structural: The structural models of texture presume that textures are combinations of texture primitives. Conceptually, structural texture analysis carried out into two major steps i.e. extraction of the texture elements, and inference of the placement rule. Two different structural methods are considered i.e. two dimensional wavelet transform and Gabor transform.

Fractals: Many natural surfaces possess a statistical quality of roughness and self-similarity at different scales. Fractals have become very useful and popular in modelling these properties in the image processing field.

Signal processing: Texture is especially suited for this type of analysis because of its properties. Includes spatial domain filters, Fourier domain, two-dimensional Gabor function.

\subsubsection{Texture feature extraction methods}

The extraction methods are used for extracting interesting and relevant features from the inputted image. The one which is used for the extraction of texture feature from images is called texture feature extraction method. The popular extraction techniques in texture field are discussed in this section.

Color co-occurrence Method: In statistical texture analysis, the texture features are computed from the statistical distribution of observed combinations of intensities at specified positions relative to each other in the image [2], [10]. Grey Level Co-occurrence Matrices (GLCM) is a statistical method. It is an old and used feature extraction method for texture classification. It has been an important feature extraction method in the domain of texture classification that computes the relationship between pixel pairs in the image. The textural features can be calculated from the generated GLCMs, e.g. contrast, correlation, energy, entropy and homogeneity. However, in recent years, instead of using the GLCM individually, is combined with other methods. Here are few other implementations of the GLCM, other than the conventional implementation e.g. one-dimensional GLCM, second-order statistical GLCM. It can be also applied on different color space for color co-occurrence matrix [12]. Spatial Gray-level Dependence Matrices (SGDM) method is a way of extracting statistical texture features. Spatial Gray-level Dependence Matrices (SGDM) includes properties viz. contrast, energy, local homogeneity, and correlation [5].

Gabor Filters: Gabor filters also popular as the Gabor wavelets. It is a widely used signal processing method. The Gabor filters consists of parameters such as the radial centre frequency, standard deviation and orientation. It can be can be used by defining a set of radial centre frequencies and orientations. As signal processing method produces large feature size, Gabor filters requires to be downsized for the prevention of the dimensionality issues [9]. Principal Component Analysis (PCA) can be a good choice to downsize the feature space Though Gabor filters are popular in texture classification it sometimes combined with other methods too [1].

Wavelets Transform: Another popularly used signal processing method in image processing and pattern recognition is wavelet transforms. Currently, it became an important feature to be used in texture classification. Several wavelet transforms are used popularly nowadays such as Discrete Wavelet Transforms (DWT), Haar wavelet and Daubechies wavelets. Among these DWT is most widely used wavelet transform. The information on the frequency domain is usually more stable as compared to the spatial domain. Therefore, despite being more complex and slower, wavelet transforms usually produces better features with a higher accuracy [3]. 
Principal component analysis: PCA is a statistical procedure that uses orthogonal transformation to convert a set of observations of possibly correlated variables into a set of values of linearly uncorrelated variables called principal components. The number of original variables is greater than or equal to the number of principal components. It is sensitive to the relative scaling of the original variables [15]. PCA is the simplest of the true eigenvector-based multivariate analyses; PCA is closely related to factor analysis [8].

\subsection{Classifier}

A software routine was written in MATLAB. In which training and testing performed via several neural network classifier. Texture Feature Classification Methods are as follows.

\subsubsection{K-nearest neighbour}

$\mathrm{K}$-nearest neighbor classifier is used to calculate the minimum distance between the given point and other points to determine the given point belongs to which class. Goal is to computes the distance from the query sample to every training sample and selects the neighbor that is having minimum distance.

\subsubsection{Radial basis function}

A radial basis function $(\mathrm{RBF})$ is a real-valued function whose value depends only on the distance from the origin. The normally used measuring norm is Euclidean distance. RBF's are the networks where the activation of hidden units is based on the distance between the input vector and a prototype vector [15].

\subsubsection{Artificial neural networks}

ANNs are popular machine learning algorithms that are in a wide use in recent years. Multilayer Perception (MLP) is the basic form of ANN that updates the weights through back propagation during the training $[1,14]$. There are other variations in neural networks, which are recently, became popular in texture classification [10], [12].

Probabilistic Neural Network (PNN): It is derived from Radial Basis Function (RBF) network and it has parallel distributed processor that has a natural tendency for storing experiential knowledge. PNN is an implementation of a statistical algorithm called kernel discriminate analysis in which the operations are organized into a multilayered feed forward network having four layers viz. input layer, pattern layer, summation layer, and output layer [15].

Convolutional neural network: It is a neural network that has convolution input layers acts as a self learning feature extractor directly from input images. Hence, it can perform both feature extraction and classification under the same architecture.

Back propagation network: A typical BP network consists of three parts: input layer, hidden layer and output layer. Three parts in turn connect through the collection weight value between nodes [11, 15]. The largest characteristic of BP network is that network weight value reach expectations through the sum of error squares between the network output and the sample output, and then it continuously adjusted network structure's weight value $[8,12,13]$. It is popular and extensively used for training feed forward networks. Also it has no inherent novelty detection, so it must be trained on known outcomes for training feed forward networks.

\subsubsection{Support vector machine}

Support vector machine (SVM) is a non-linear classifier, and is a newer trend in machine learning algorithm. SVM is popularly used in many pattern recognition problems including texture classification [14]. SVM is designed to work with only two classes. This is done by maximizing the margin from the hyper plane. The samples closest to the margin that were selected to determine the hyper plane is known as support vectors. Multiclass classification is applicable and basically built up by various two class SVMs to solve the problem, either by using one-versus-all or one [10].

Table.1. Texture classification techniques comparison

\begin{tabular}{|c|l|l|l|}
\hline $\begin{array}{c}\text { Sr } \\
\text { No }\end{array}$ & \multicolumn{1}{|c|}{ Technique } & \multicolumn{1}{|c|}{ Advantages } & \multicolumn{1}{c|}{ Disadvantages } \\
\hline 1 & $\begin{array}{l}\text { K-Nearest } \\
\text { Neighbor } \\
(\text { KNN) }\end{array}$ & $\begin{array}{l}\text { Simpler classifier as exclusion of any } \\
\text { training process. } \\
\text { Applicable in case of a small dataset } \\
\text { which is not trained. }\end{array}$ & $\begin{array}{l}\text { Speed of computing distance increases } \\
\text { according to numbers available in training } \\
\text { samples. } \\
\text { Expensive testing of each instance and } \\
\text { sensitive to irrelevant inputs. }\end{array}$ \\
\hline 2 & $\begin{array}{l}\text { Radial Basis } \\
\text { Function } \\
\text { (RBF) }\end{array}$ & $\begin{array}{l}\text { Training phase is faster. } \\
\text { Hidden layer is easier to interpret. }\end{array}$ & $\begin{array}{l}\text { It is slower in execution when speed is a } \\
\text { factor. }\end{array}$ \\
\hline 3 & $\begin{array}{l}\text { Probabilistic } \\
\text { Neural } \\
\text { Networks }\end{array}$ & $\begin{array}{l}\text { Tolerant of noisy inputs. } \\
\text { Instances classified by more than one } \\
\text { output }\end{array}$ & $\begin{array}{l}\text { Long training time. } \\
\text { Large complexity of network structure. } \\
\text { Need lot of memory for training data. }\end{array}$ \\
\hline
\end{tabular}




\begin{tabular}{|l|l|l|l|}
\hline & (PNN) & Adaptive to changing data. & \\
\hline 4 & $\begin{array}{l}\text { Back- } \\
\text { propagation } \\
\text { Network } \\
\text { (BPN) }\end{array}$ & $\begin{array}{l}\text { Easy to implement. } \\
\text { Applicable to wide range of problems. } \\
\text { Able to form arbitrarily complex } \\
\text { nonlinear mappings }\end{array}$ & $\begin{array}{l}\text { Learning can be slow. } \\
\text { It is hard to know how many neurons as } \\
\text { well as layers are required. }\end{array}$ \\
\hline 5 & $\begin{array}{l}\text { Support } \\
\text { Vector } \\
\text { Machine } \\
\text { (SVM) }\end{array}$ & $\begin{array}{l}\text { Simple geometric interpretation and a } \\
\text { sparse solution. } \\
\text { Can be robust, even when training } \\
\text { sample has some bias. }\end{array}$ & $\begin{array}{l}\text { Slow training. } \\
\text { Difficult to understand structure of } \\
\text { algorithm. Large no. support vectors are } \\
\text { needed from training set to perform } \\
\text { classification task. }\end{array}$ \\
\hline
\end{tabular}

\section{Conclusion}

The present paper reviews and summarizes image processing techniques for several plant species that have been used for recognizing plant diseases. The major techniques for detection of plant diseases are: BPNN, SVM, K-means clustering, and SGDM. These techniques are used to analyses the healthy and diseased plants leaves. Some of the challenges in these techniques viz. effect of background data in the resulting image, optimization of the technique for a specific plant leaf diseases, and automation of the technique for continuous automated monitoring of plant leaf diseases under real world field conditions. The review suggests that this disease detection technique shows a good potential with an ability to detect plant leaf diseases and some limitations. Therefore, there is scope of improvement in the existing research.

\section{REFERENCES}

[1] Anand H. Kulkarni, Ashwin Patil R. K., Applying image processing technique to detect plant diseases, International Journal of Modern Engineering Research, vol.2, Issue.5, pp: 3661-3664, 2012.

[2] F. Argenti,L. Alparone,G. Benelli ," Fast algorithms for texture analysis using co-occurrence matrices" Radar and Signal Processing, IEE Proceedings, vol. 137, Issue 6, pp:443-448, No. 6, December 1990

[3] P. Revathi, M. Hemalatha, Classification of Cotton Leaf Spot Diseases Using Image Processing Edge Detection Techniques, IEEE International Conference on Emerging Trends in Science, Engineering and Technology, pp-169-173, Tiruchirappalli, Tamilnadu, India, 2012.

[4] Tushar H. Jaware, Ravindra D. Badgujar and Prashant G. Patil, Crop disease detection using image segmentation, National Conference on Advances in Communication and Computing, World Journal of Science and Technology, pp:190-194, Dhule, Maharashtra, India, 2012.

[5] Prof.Sanjay B. Dhaygude, Mr.Nitin P. Kumbhar, Agricultural plant Leaf Disease Detection Using Image Processing, International Journal of Advanced Research in Electrical, Electronics and Instrumentation Engineering , S \& S Publication vol. 2, Issue 1, pp: 599-602, 2013.

[6] Mokhled S. Al-Tarawneh An Empirical Investigation of Olive Leave Spot Disease Using Auto-Cropping Segmentation and Fuzzy C-Means Classification, World Applied Sciences Journal, vol.23, no.9, pp:1207-1211,2013.

[7] Yan-Cheng Zhang, Han-Ping Mao, Bo Hu, Ming -Xi Li, Feature Selection of Cotton Disease leaves Image Based on Fuzzy feature Selection Techniques, Proceedings of the 2007 International Conference on Wavelet Analysis and Pattern Recognition, pp:124-129, Beijing, China, Nov. 2007.

[8] Haiguang Wang, Guanlin Li, Zhanhong Ma, Xiaolong Li, Image Recognition of Plant Diseases Based on Back propagation Networks, 5th International Congress on Image and Signal Processing, pp-894-900, Chongqing, China, 2012

[9] Simona E. Grigorescu, Nicolai Petkov, and Peter Kruizinga, Comparison of Texture Features Based on Gabor Filters , IEEE ransaction on Image Processing, vol. 11, No. 10, pp-1160-1167, oct 2002.

[10] S. Arivazhagan, R. Newlin Shebiah, S. Ananthi, S. Vishnu Varthini, Detection of unhealthy region of plant leaves and classification of plant leaf diseases using texture features, Commission Internationale du Genie Rural(CIGR) journal, vol. 15, no.1, pp:211-217, March 2013.

[11] Dheeb Al Bashish, M. Braik, and S. Bani-Ahmad, A Framework for Detection and Classification of Plant Leaf and Stem Diseases, 2010 International Conference on Signal and Image Processing, pp: 113-118, Chennai, India, 2010.

[12] Song Kai, liu zhikun, Su hang, Guo chunhong, A Research of maize disease image recognition of Corn Based on BP Networks, Third International Conference on Measuring Technology and Mechatronics Automation,pp:246-249, Shenyang, China, 2011.

[13] H. Al-Hiary, S. Bani-Ahmad, M. Reyalat, M. Braik and Z. ALRahamneh, Fast and Accurate Detection and Classification of Plant Diseases, International Journal of Computer Applications, Wageningen Academic publishers, vol. 17, no.1, pp: 31-38, March 2011.

[14] A.Menukaewjinda, P.Kumsawat, K.Attakitmongcol, A.Srikaew, Grape leaf disease detection from color imagery using hybrid intelligent system, Proceedings of electrical Engineering/electronics, Computer, Telecommunications and Information technology (ECTI-CON), vol 1. pp: 513-516, Krabi, Thailand, 2008.

[15] Haiguang Wang, Guanlin Li, Zhanhong Ma, Xiaolong Li , Image Recognition of Plant Diseases Based on Principal Component Analysis and Neural Networks, 8th International Conference on Natural Computation, pp-246-251, Chongqing, China, 2012 . 